-ORTIONS OF THIS REPORT $\quad \therefore \ldots \ldots$

5iecr reproduced from the bses a

L:CRI.-90494

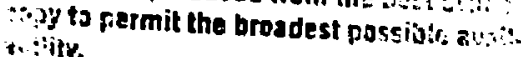

PREPRINT

A. AF- $-4 \times 7-2$

\title{
Developments of Optical Fast-Gated Imaging Systems
}

\author{
H. A. Koehler and D. E. Kotecki
}

This paper was prepared for the Proceedings of the 16th International Congress on High Speed Photography and Photonics

Strasbourg, France

August 27-31, 1984

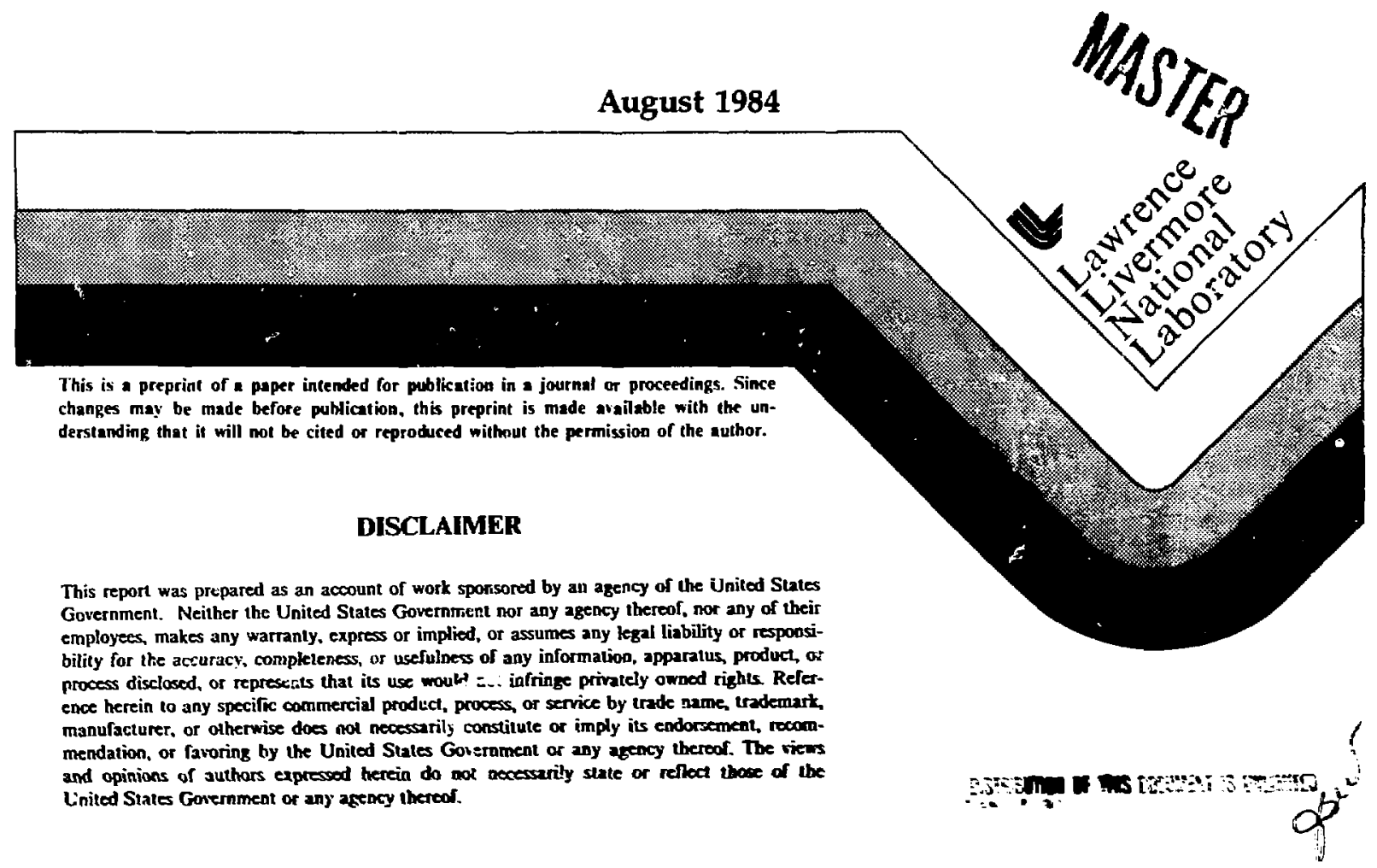




\title{
Developments of optical fast-gated imaging systems
}

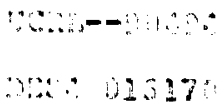

I1. A. Kixhler and D. Kotrock:

Lik rence Livermore National Laboratory; University of California

P.O. Box ROB, Livermors, California 94550

\begin{abstract}
Ábstract
Several fast-gated imaging systems to measure ultra-fast single-transient data have been developed for time-resolved imaging of pulsed radiation sources. These systems wern designed to achieve image recording times of $1-3 \mathrm{~ms}$ and dynamic ranges of $>200: 1$ to produce large (wo-4jimensional images ( $\geq 10^{1}$ sputial points) of 1-2 ns exposure and small two-dimensional images ( $\geq 200$ spatial points) of $\leq 0.5 \mathrm{~ns}$ ixposure. Both MCP inten:sified solid-stak two-dimensional framing cameras and streak camera/solid-state camera systems were used the framing camera system provides snap shots with high spatial resolution whereas the streak camera system provides for limited spatial point, each with high tempora! resolution. Applications of these systems include electron-beam, $x$-ray, gamma-ray, and neutron diagnostics. This repurt reviews the characieristics of the major components of fast-gated imaging systems developed at Lawrence Livermore National Laboratory. System perfornances are described in view of major experiments, and the diagnostic requirements of new experiments in atomic physics ( $x$-ray lasers) and nuclear physics (fusion) are indicated.
\end{abstract}

\section{Intruduction}

Several fast-gated imaging systems have been investignted to measure ultra-fast single-transient data from pulsed radiation sources. Work on these systems was stimulated by increased demand at Lawrence Livermore National Laboratory (LLNL) for diagnostic systems of this type. These systems were designed to achieve the following criteria:

- Image recording time into memory of $1-3 \mathrm{~ms}$ and

- Broad sensitivity/dynamic range of $>200: 1$ to produce:

- Large two-dimensional images ( $\geq 10^{4}$ spatial points) of 1-2 ns exposure and

- Small two-dimensional images ( $\geq 2$ vius spatial points) of $\leq 0.5 \mathrm{~ns}$ exposure.

Generally, these imaging systems are sophisticated pinhole cameras using a pinhole or slit to focus $x$ rays, gamma rays, or neutrons onto a convarter (fluor); visible light from the fluor is focused onto the photosensors of the recording camera. Fast shuttering of these images is provided by both gated microchannel-plate (MCP) intensifiers and streak cameras that are placed on frort of the recording cameras. The recorded im ages are digitized and stored in nonvolatile memories. Physically meaningful pictures are obtained after computer processing of the experimental and calibration data.

Applications of these systems include electron-beam, $x$-ray, gamma-ray, and neutron diagnostics. Electron-beam diagnostics are used to provide information about the size and position of the beam, currents in the beam, and its propagation characteristics. $X$-ray diagnostics are used to make time-resolved $x$-ray measurements. Gamma-ray and neutron diagnostics include electronic pinhole neutron expuriments and tomography, which are used to study burn efficiencies and implosion symmetries af fission/fusion devices.

This report reviews the characteristics of the majo- components of fast-gated imaging systems that have been developed in the Nuclear Test Program of LLNL. The system performunces are described in view of major experiments.

\section{Imaging system components}

The major components of a gated imaging system are the shuiter, the camera, and the data acquisition system. The shutter m.ay be a MCP intensifier, a streak camera, or an internal gating grid. The camera may be either a solid-state Reticon using $100 \times 100$. $256 \times 256$, or $1024 \times 1$ photodiode arr.⿲y sensors, a Fairchild camera using $488 \times 380$ charge-colpled device (CCD), or a vacuumtube Ceneral Electric FPS-GSIT (focus-projection-scan: gated silicon intensified target) vidicon camera. The digital data acquisition systems used for all cameras consist of memories, computers, and recorders.

\section{Opiral shutters}

Gated MCP intensifiers and streak cameras have been examinesi extensively as uptical shutters of fast-framing cameras. ${ }^{1-4}$

MCP intensifiers. The MCP intensifier consists of a phntocathode (PC), a MCP electron multiplier, and a phosphor sereen that constitutes the photon source viewed by the camera. The photocathode is proximity focused $(\sim 0.25 \mathrm{~mm})$ onto the MCP $\left(\sim 10^{\overline{7}}\right.$ electron gain), which is in turn proximity fccused ( $\sim 1.25 \mathrm{~mm}$ ) onto the phosphor screen. Optical shuttering is achieved by applying a voltage pulse between the PC and MCP or across the MCP. The MCP intensifier is gated by dc reverse-biasing and by pulse forward-biasing. Both coaxial termination geometry and microstrip transmission-line geometry are used. ${ }^{1,4}$ 


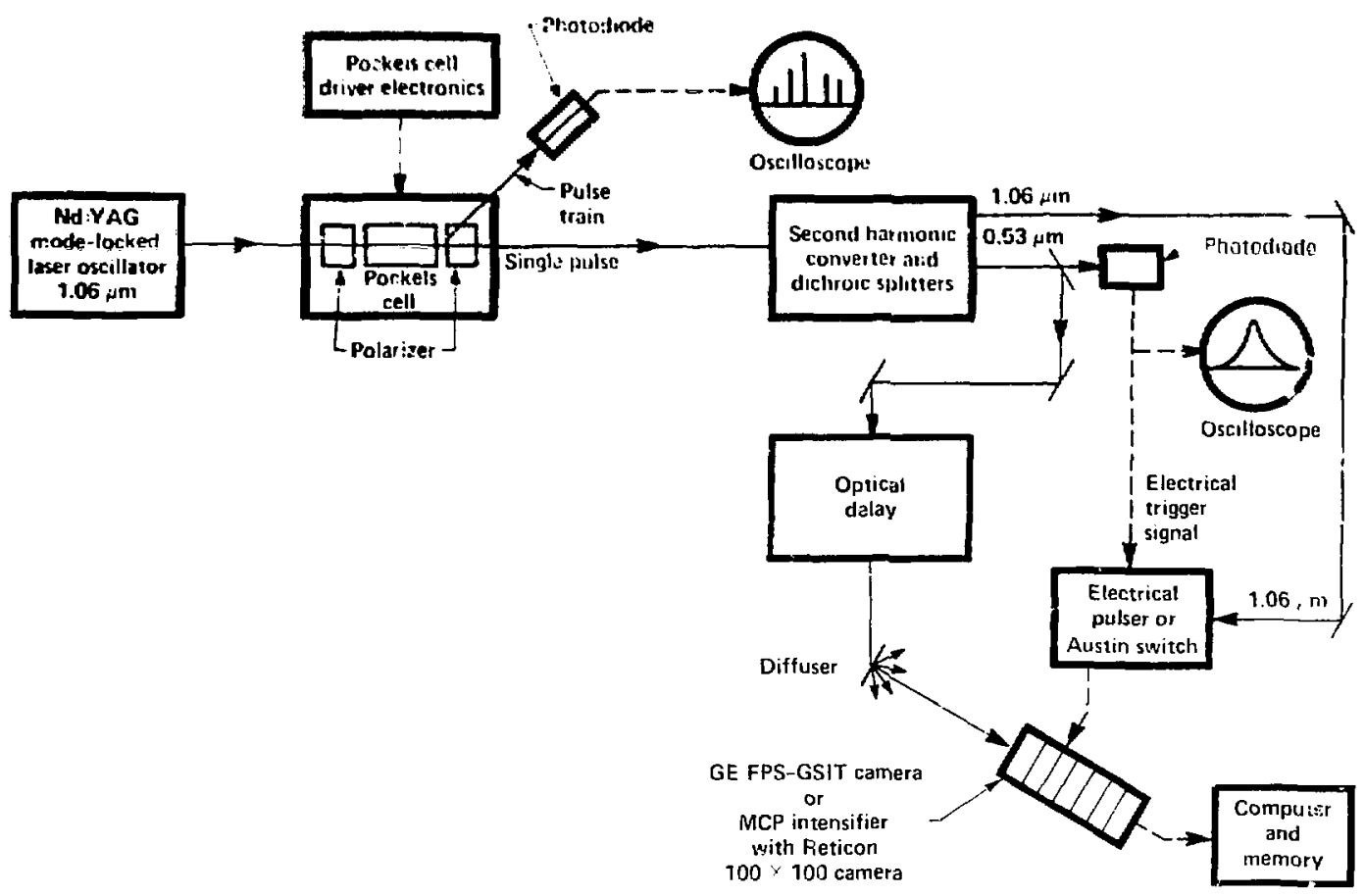

Figure 1. Calibratun arrangement for correlating vplical shuller speded to spalial resolution.

for most high-speed imaging applications, the correlation of epticil shutter speed to spatial resolution at a fixed cont rast transfer tunction (CTF) must be precisely . rmined. Figure 1 shows a typical calibratuen asrangement. A single nulse from a mede-kicked

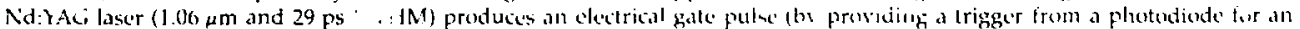
avalanche transistor pulser or by energizing a photocenductive swith). A purtin of the oiginal pulse is trequency-soubled at $532 \mathrm{nn}$; this pulse travels through ..n optial delay line of $70-140 \mathrm{~m}$ (adjustable in 50 -ps steps) onto a diffuser. A MClP intensifier

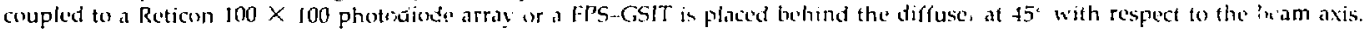
The opticat shutter length is measured be increasing the optical delay of the frequency-doubled laser pulse in relation to the fixed electrical gating pulse. The digital output of tive camers is sent to a mincemputer and recordes on a floppy disk rer image' processing.

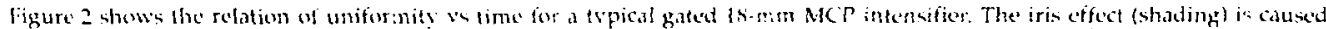

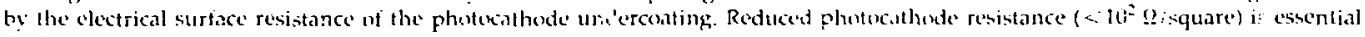
for fast gating.
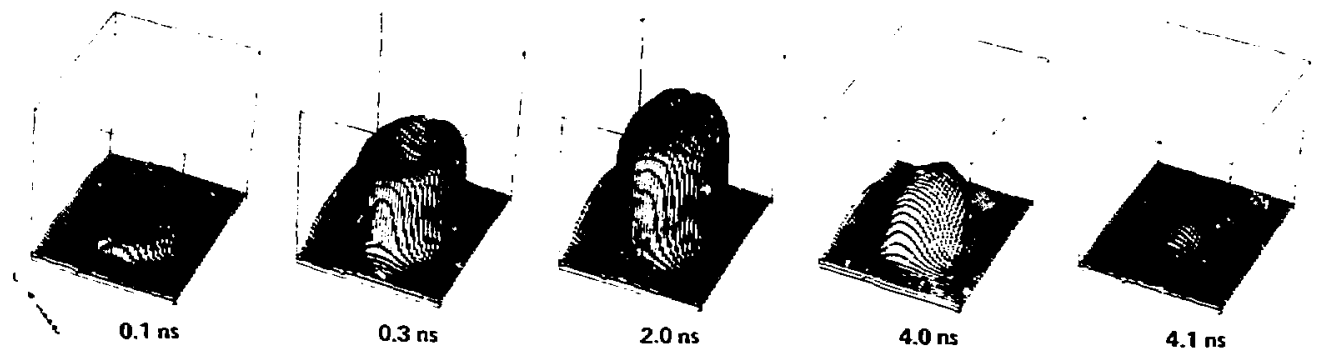

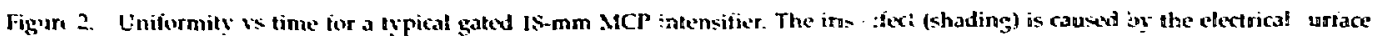
resistunce of the phutexathente undercenting. 


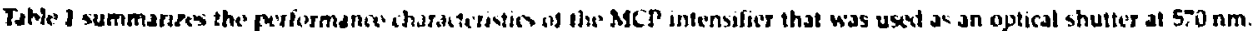

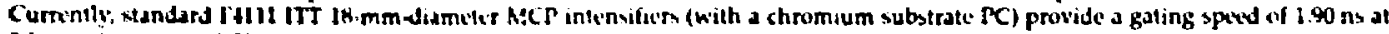

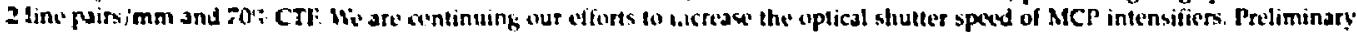

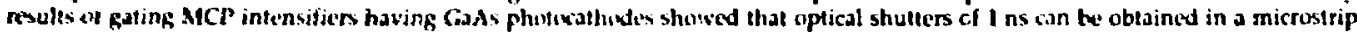
transmission-line gerometry. Experiments with wrtur gating. segmented $\Gamma$ s, and with triple-thickness PCs at Los Alamos National Lborstory (Los Alamos, NA) increased the' gating sperd to 1 .5 ns."

Table 1. Performance characteristics of the MCP intensifier and the s'reak camera.

\begin{tabular}{|c|c|c|c|}
\hline Chura-teristic & Goal & MCP intensificr & Streak camera \\
\hline Shutter speed (ns) & $\ll 1.0$ & $1.9(<1.0\}^{\mathrm{c}}$ & $0.05-500^{\mathrm{s}}$ \\
\hline Rexolution (ip/nm) & $B$ & 2 & 4 \\
\hline Resolvable points & 100,000 & $10,000^{:}$ & 250 \\
\hline Extinction ratio & $10^{6}: 1$ & $>10^{6}: 1$ & $10^{6}: 1$ \\
\hline Dynamic range & $>200: 1$ & $200: 1^{8}$ & $100: 1$ \\
\hline Gain (radiometric) & $10^{4}$ & $10^{4}\left(10^{3}\right)^{1}$ & 500 \\
\hline Temporal somples & $>1$ & 1 & $380^{\mathrm{h}}$ \\
\hline Satiuration $\left(\mathrm{erg} / \mathrm{cm}^{2}\right)$ at $570 \mathrm{~nm}$ & - & $10^{-3}$ & $10^{8}-10^{4} / 5^{1}$ \\
\hline
\end{tabular}

a $18-\mathrm{mm}$ diameter.

b With 40-mm-diameter MCP intensifier.

'GaAs pilotocathode.

$0.5 \%$ of sweep time, where sweep time is $0.01-100 \mu \mathrm{s}$

"At 70\% CTF.

With Reticon $100 \times 100$ camera.

8 Using 8-bit digitizer.

h Fairchild $244 \times 380$ CCD.

i Corresponding to a sweep time of $0.01-160 \mu \mathrm{s}$.

We are currently designing a portable electrical pulser that produces $>300 \mathrm{~V}$ with a pulse width of $<0.5 \mathrm{~ns}$ (witi $<0.1 \mathrm{~ns}$ jitter) into an impedance load of $17 \Omega \Omega^{7-9}$ Specially packaged step recovery diodes and photoconductive switches driven by laser diodes are cons dered in these designs. ${ }^{4.5}$

Streak cameras. Streak cameras are optical shutters; they convert a pulse of light from a point source into a streak whose points correspond to the temporal sequence of the light intensity. Light strikes the photoca:hode througin a slit and generates a pulse of photoelectrons that is accelerated toward a phosphor screen. The electrons are deflected by sweep electrudes and spread out on the phosphor screen. Ger:erally, the screen is viewed by a camera through a MCP intensifier.

The streak camera described in this report was built around the RCA 73435 AK and AJ series tube. ${ }^{l}$ This tube is a gated e iectrustatic-focus, electrostatic-deflection image device that is specifically designed for high-speed light shutters. The image tube has a iiter-optic or glass input window and a fiber-optic output window, a modified S-20 photocathode (allowing extended UV and IR res jonse), and a P-11 phosphor. This tube was chosen because of its relatively large PC $(60 \times 25 \mathrm{~mm})$, which permits 250 resolvable linus at 70\% CTF. Single photoelectrons can be observed at the phosphor. The extraction grid is pulse-biased to improve the extinction ratio (to $\sim 10^{6}$ ). A 40-mm-diameter ITT MCP intensifier having an S-20 PC and a P-20 phosphor coupled to the streak tube by fiber optics provides a sensitivity adjustment as well as a better spectral match to the silicon response of the solid-state recording cameras. Adding a MCP intensifier to the streak camera, however, degrades the spectral resolution. For instance, at $70 \%$ CTF and $150 \mathrm{~ns}$ sweep, the spatial resolution with and without the MCP intensifier is 4 and $5.5 \mathrm{lp} / \mathrm{mm}$, respectively. Table 1 summarizes the streak-camera performance

We are currently investigating the feasibility of replacing the RCA streak tube with one of increased quantum efficiency at $820 \mathrm{~nm}$ and larger spatial resolution. Development work is currently being conducted by both ITT Electro-Optics Products Division (Fort Wayne, IN) and EGd $G$, inc. (San Ramon, $C_{A}$ ) to meet these higher performance goals.

\section{Cameras}

We have examined three kinds of cameras: solid-state Reticon and Fairchild cameras, and vacuum-tube GE FIS-GSIT vidicon came:as.

We used a modified TV Optoliner (Optical Instruments Corp.) and a pulsed light source to measure the linearity to incident light, dynamic range, spatial uniformity, and absolute sensitivity for each camera at a fixed wavelengtin. The output energy of the Optoliner was measured using a calibrated photodiode. Calibrations of the cameras were performed by averaging the output of all pixels for each Optoliner pulse taken at different intensity levels.

Reticon cameras. The main components of the Retico; solid-state camera system are the camera controlier, the camera head (containing the sensor and preamptifier), and the power supply. The controller contains the amplifier, digitizer, and clocks. 


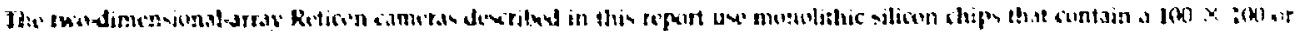

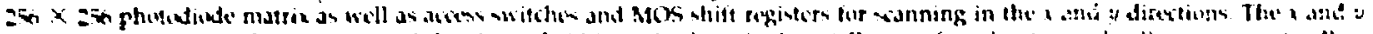

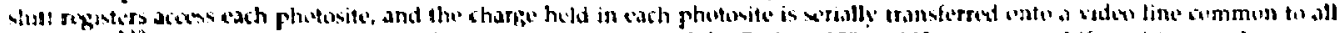

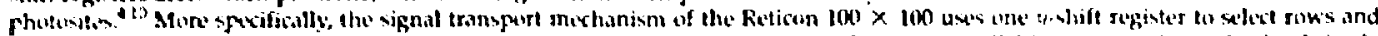

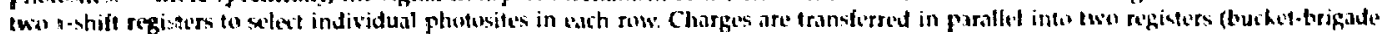

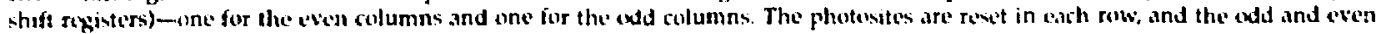
solumn chages are clocked to serial output.

Reticon $100 \times 100$. Wh measuret the linearity, dynamic range, sesolution, and siturateon of this camera (Table 2). The' array size is $6 \times 6 \mathrm{~mm}$, the celiter-to-center spacing of the photosites is $60 \mu \mathrm{m}$, and the aclive area is $56^{\circ} ;$ of the array size:

The camera was designed at LLNL. Variations from commercially available cameras unctuded decreusine, the read-(1)ut rate to $2.5 \mathrm{~ms} /$ frame, digitizing the video output signal, and repackaging the camera for operating in an adverse covironment. The cameras were designed to be reset on receiving a trigger signal, thus allowing them to record pulsed light images. Also, the absence of automatic gain control enabled the camera to be used as a diagnostic of absolute enerby measurements. The Reticon $100 \times 100$ array can be outfitted with a fiber-optic faceplate to allow for fiber-optic coupling of a MCP intensifier tube.

Reticon $256 \times 256$. We are also presently testing a new camera that provides larger spatial resolution. "This c.3mera uses a Reticon $256 \times 256$ photodiode array sensor. The array size is $10.24 \times 10.24 \mathrm{~mm}$, the center-to-center spacing of the photosites is $40 \mu \mathrm{m}$, and the active area is $63 \%$ of the array size. Table 2 summarizes the performance goals. Saturation sensitivity of $\sim 1.5 \mathrm{erg}^{2} \mathrm{~cm}^{2}$ at $570 \mathrm{~nm}$ is desired since most solid-state cameras are coupled to MCP intensifiers that have a maximum output of $\sim 1.2 \mathrm{erg} / \mathrm{cm}^{2}$.

The architccture of the array consists of eight contiguous blocks of $32 \times 256$ photosires, each haviug its own 5 migapixel video output. With additional multiplexing of two video lines each, a frame time of $1.8 \mathrm{~ms}$ is expected

Reticon $1024 \times 1$. A Reticon $1024 \times 1$ linear array of silicon photodiodes is used with spectrometers in high-speed diagnostic systems. Fhe array size is $12.5 \mu \mathrm{m} \times 2.5 \mathrm{~mm}$, the center-to-center spacing of the photosites is $25 \mu \mathrm{m}$, and the active area is $50 \%$ of the array size. Table 2 summarizes the characteristics of this camera. ${ }^{12}$

Fairchild camera. The solid-state Fairshild camera described here was designed at LLNL. ${ }^{13.14}$ It uses 380 horizontal and 488 vertical charge-coupled photoelements. The dimensions of the array are $8.8 \mathrm{~mm}$ horizontally and $11.4 \mathrm{~mm}$ vertically. Center-to-center spacings are 30 and $18 \mu \mathrm{m}$ in the horizontal and veltical directions, respectively. The active area is $40 \%$ of the array size.

Photoelectrons collected in the photosites are transferred first from the odd-numbered rows to a vertical transport register, then to a horizontal transport register. The even-numbered phatosite rows are read out next in a similar manner. To adapt this camera to a high-speed diagnostic system, every two consecutive rows are combined (on-chip addition, OCA) and the clocking rate of the array is increased. The analog video output is digitized at $20 \mathrm{MHz}(8 \mathrm{bits})$. This noninterlaced, one field/frame format provides a frame time of $5 \mathrm{~ms}$. Table 2 lists some of the characteristics of this camera. The dynamic range of the camera is limited by the 8-bjt digitizer to 256:1. Adjusting the camera gain for increased sensitivity decreases the dynamic range. Contrary to the Reticon photodiode arrays, the CCD sensors exhibit "blooming" at $-3 \times$ saturation value in the vertical direction only.

Figure 3 shows a typical response of a gated 2.6-ns MCP intensified Fairchild $244 \times 380$ camera to a uniform optical field of varying intensity from the Optoliner. (Figure 3 indicates three typical flat-field responses.) A least-squares fit was Ferformed on the discrete data points. The camera saturated at $1.2 \mathrm{merg} / \mathrm{cm}^{2}$ using $570-\mathrm{nm}$ light. The standard deviation of the recorded output from all photosites is shown as vertical bars; 30 counts out ut 223 counts was the worst case. This includes the shading inlierent in the pulsed light source. The tw' 1 l operating range of 220 counts is determined by the hackground (lower limit) and the digitizer (upper limit). Generally, fixed-pa.rern noise and random fluctuations are less than $0.5 \%$ of the saturation level.

Table 2. Performance characteristics of Reticon cameras and the Fairchild CCD.

\begin{tabular}{|c|c|c|c|c|c|}
\hline Characteristic & Goal & Retico & photodiode & array & Fairchild CCD \\
\hline $\begin{array}{l}\text { Pixels } \\
\text { Resolution (lp/mm) } \\
\text { Frame time (ms) } \\
\text { Dynamic range } \\
\text { Saturation (erg/cm } \\
\text { Digitizer (bit) } \\
\text { Field uniformity }\left(\mathrm{cm}^{\mathrm{c}}\right)^{\mathrm{d}}\end{array}$ & $\begin{array}{l}10^{5} \\
15 \\
<2 \\
>200: 1 \\
<1.5^{\mathrm{h}} \\
10 \\
i>0\end{array}$ & $\begin{array}{l}100 \times 100 \\
8 \\
2.5 \\
500: 1 \\
1.7(0.002)^{e} \\
8 \\
>95\end{array}$ & $\begin{array}{l}256 \times 256 \\
12^{\mathrm{b}} \\
1.8^{\mathrm{b}} \\
500: 1^{\mathrm{b}} \\
1.5^{\mathrm{b}} \\
9^{\mathrm{b}} \\
>95^{\mathrm{b}}\end{array}$ & $\begin{array}{l}1024 \times 1 \\
7 \\
1 \\
1000:: \\
0.8 \\
10 \\
>95\end{array}$ & $\begin{array}{l}244 \times .350 \\
6 \times 8 \\
5 \\
750: 1 \\
0.9(0.001)^{e} \\
8 \\
>95\end{array}$ \\
\hline
\end{tabular}

At 70\% CTF.

Design goals.

'Photosensor only; dynamic range is maximum signal/RMS noise.

At $5 r 0 \mathrm{~nm}$.

With $M C P$ intensifier (fiber-optic input).
Table 3. Performance characteristics of the GE FPS-GSIT vidicon camera.

\begin{tabular}{lc}
\hline \multicolumn{1}{c}{ Characteristic } & $\begin{array}{l}\text { GE FPS-GSIT } \\
\text { vidicon camera }\end{array}$ \\
\hline Pixels & \multicolumn{1}{c}{$470 \times 256^{\mathrm{b}}$} \\
Resolution $(1 \mathrm{p} / \mathrm{mm})^{\mathrm{c}}$ & 5 \\
Frame time $(\mathrm{ms})$ & $\geq 1.6$ \\
Dynamic range & $\geq(50-200): 1$ \\
Saturation $\left(\mathrm{erg} / \mathrm{cm}^{2}\right)^{\mathrm{e}}$ & $\mathbf{0 . 0 1}$ \\
Digitizer (bit) & $\mathbf{8}$ \\
Field uniformity (๕) & $\leq 75$ \\
Gating speed (ns) & $\geq 0.5$ \\
Extinction ratio & $(60-1000): 1$ \\
\hline
\end{tabular}

25-mm-diameter S-20 phctocathode.

3.2-ms read-out.

At 70\% CTF with i to 2-ns gate.

'Photosensor only.

At $570 \mathrm{~nm}$. 


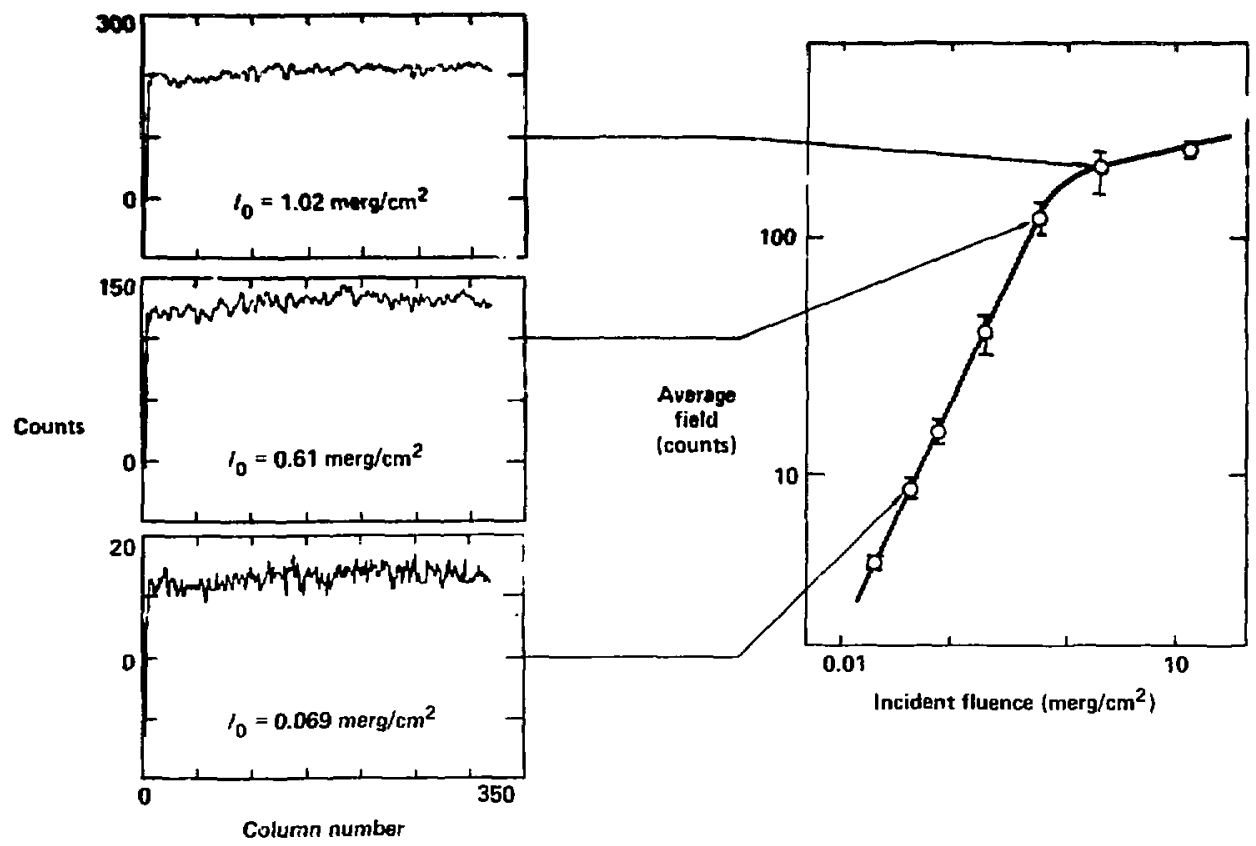

Figure 3. Response of a gater MCP intensified Fairchild $244 \times 380$ camera to a uniform optical field of varying ir ensity. The optical gate was $2.6 \mathrm{~ns}$ and the calibration wavelength was $570 \mathrm{~nm}$. Three typical flat-field responses are shown.

GE FPS-GSIT vidicon camera. The GE FPS-GSIT vidicon camera consists of a camera head and a contr unit. This camera is the result of extensive development at Los Alamos National Laboratory. ${ }^{5,6}$ The camera head contains the image sensor, amplifiers, biasing and deflection circuits. The controller contains power supplies, video processing and shading correction circuitry. The camera was designed to reset to the beginning of the field upon receiving a trigger signal. The deflection circuits are reactivated after an adjustable delay during which the cathode is blanked. The time delay is used to integrate the pulse image on the sensor so that the entire pulse is received before read-out of the sensor begins. The image format is 256 scan lines/field, noninterlaced. The read-out can be varied from 1.6 to $25.6 \mathrm{~ms}$. A $40-\mathrm{M} 4 \mathrm{~Hz}$ digitizer and a 1.6 -ms frame time provim-s an image size of 236 columns by 256 ows. For a 3.2 -ms frame time, the number of rolumns is increased to 472 .

The FPS-GSIT can be gated to produce optical shutters with durations as short as $=00$ ps. Photoelectrons from the S-20 phutocathode pass through the gating grid and are electrostatically focused onto the other side of the silicon target. The video output signal is proportional to the beam current necessary to recharge the silicon target diodes to the cathode potesitial.

The optical shutter speed of the FPS-GSIT vidicon was recenily measured using the RF LINAC at the EG\&G facility in Santa Barbara, CA. A Faraday cup in the LINAC beam produced a 200-ps FWHM gating pulse that resulted in an optical shutter of $\sim 500$ ps FWHM. Because the phntocathode was coated after installation into the tube envelope near the gating grid, the measured extinction ratio was only $\sim 60: 1$. 'The extinction ratio is defined as the ratio of light intensities that, when focused onto the PC when the t'be is gated off and on, producr the same output signal.) Table 3 summarizes the performance characteristics of the FPS-CSIT vidicon camera.

Comparison of cameras. The Reticon $100 \times 100$ photodiode array and the Fairchild $244 \times 380$ CCD solid-state cameras have been used extensively at LLNL for high-speed inaging. The Reticon $1024 \times 1$ linear array has been used only rezently with spectrometers. The Reticon $256 \times 256$ is still under development. Some applications of these cameras are discussed btlow.

The advantages of the MCP intensified solid-state comeras over vacuum-tube FPS-GSIT vidicon cameras are excellent field uniformity and linearity, small geometric distortion, relatively large linear dynamic range, a large extinction ratio, anu compactness. The disadvantages are relatively slaw optical gating speed and low spatial resolution.

The advantages of the vacuum-tube FPS-GSIT camera over the MCP intensified solid-ctate cameras are fast gating speed and a large photocathoutr. which f.ovides excellent spatial resolution. Disadvantages are low linerr dynamic range, poor field uniformityshuding. geometric distortion. low shutter ratio, and larger size. Work is in progress at Los Alamos National Laboratory to improse 


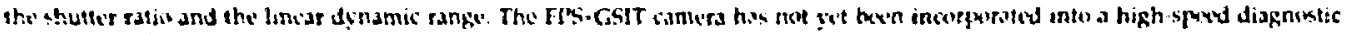
syetiom al 1 - $N$ il.

\section{Data aciguisition system}

The Reticun 160 \} 1 0 0 \text { photodiode array canteras use a data acquisition system designed at LLNL. (Fig. 4). The bit/frame } synchronizing module deserializes the $45 \cdot \mathrm{Mbi}$ / $/$ serial Bio-M PCM data stream from the $100 \times 100$ Reticon camers The memory is a dual-port, 16K, 9-bit static KAM. The unit memnry controller (UMC) is a minicomputer (LSI 11/23) that controls the datis transfer to permanent storage. Local processing of the data with the minicomputer includes background sibblraction, intensity profiles, histograms, contour plots, and three-dimensional isometric plots.

The Reticon $256 \times 256$ and $1024 \times 1$ photodiode-artay comera and the Fairchild $380 \times 488$ CCD camera use similar data acquisition systems (Fig. 4). A real-time image processor (Recognition Concept lnc.) is used in conjunction with a minicomputer to transfer chta to the nonvolatile memiries and to perman:nt storage. This rinicomputer purforms the same image processing functions avaluble for the Reticon $100 \times 100$, with the addition of pseudo-color enhancement and image magnification.

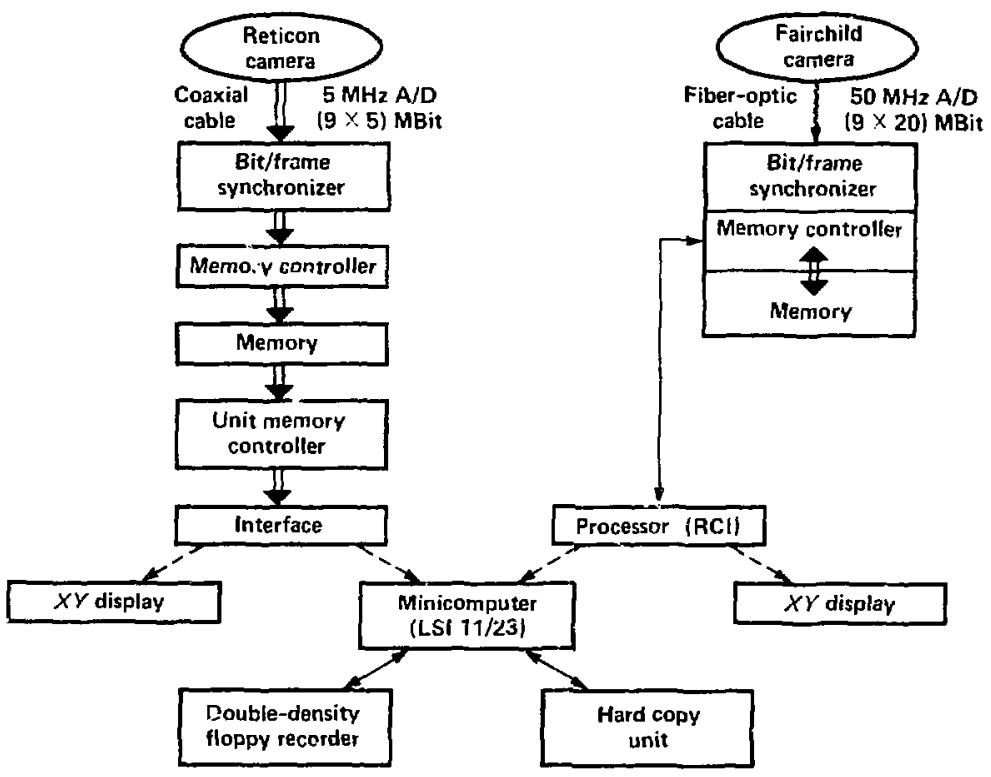

Figure 4. Data acquisition systems used by the Reticon photudiode array and Fairchild CCD cameras.

\section{Imaging system applications}

High-speed optical imaging systems are used in the Nuclear Test Program at LLNL with pulsed radiation sources in the laboratory and in the field, on accelerators, lasers, and nuclear tests. In general, MCP-gated solid-state framing cameras provide one snapshot of an event with high spatial resolution whereas streak samera/solid-state camera system provide high temporally resolved images with limited spatial resolution.

\section{Applications using MCP and solid-state cameras}

The Reticon and Fairchiid solid-state cameras are coupled to a MCP intensifier $(18,25$, or $40 \mathrm{~mm}$ in dizmeter) with a fiber-optic reducer. The coupling and interfaces greatly affect the gain and spatial resolution of the camera system. For instance, the spatial resolution for a camera with and without a MCP intensifier differs by as much as $40 \%$. Also, an increase in $\mathrm{MCCP}$ intensifier gain reduces the dynamic range and saturation value of the camera system (i.e., decreases the slope of the linearity curve, Fig. 3). For this reason, all cameras are calibrated with and without a MCP intensifier to assure proper coupling between the components.

Electron-beam diagnostics. Relativislic electron-beam cross sections and beam curnents are meacured by focusing Cerenkov radiation from thin piastic foils onto fiber-optic arrays that are coupled to gated MCP intensifiers. Cerenkov foils are used as beam current monitors if the plasma curreal contributes significantly to the air hlorescence. 


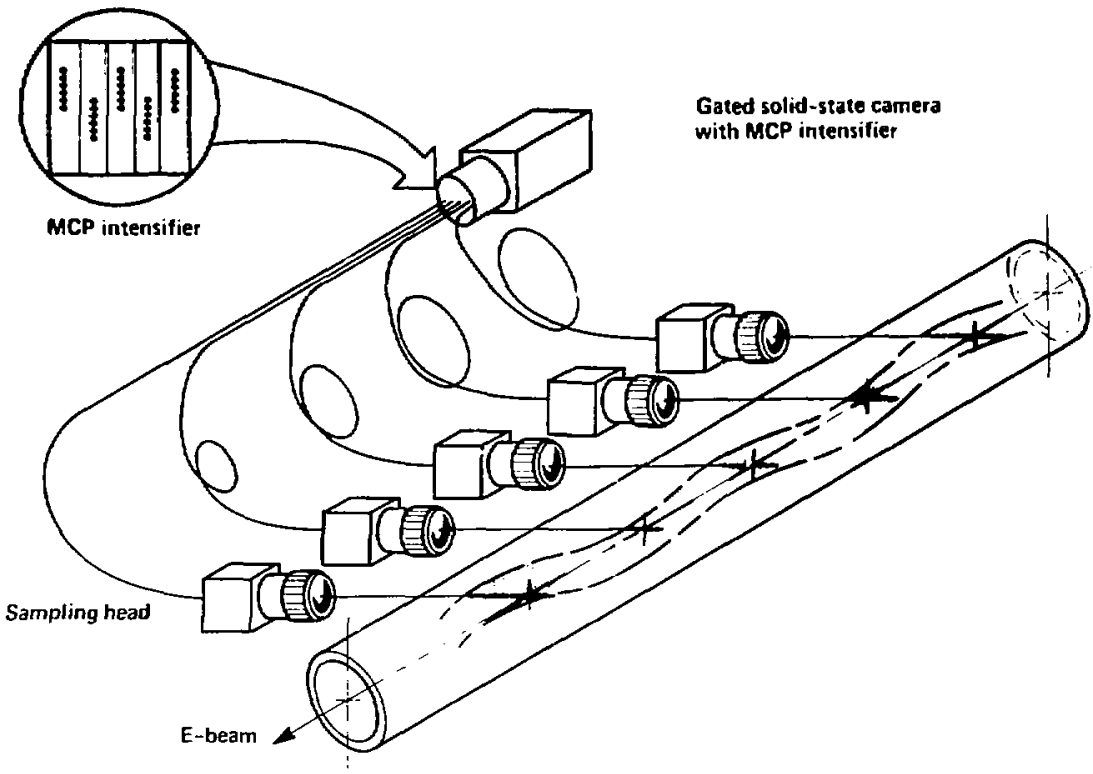

Figure 5. Fxperimental configuration for observing electron-beam propagation instabilities in a drift tube

To obserse electron-beam propagation instabilities in a drift tube the air fluorescence is focused onto fiber-optic arrays that are placed along the drift-tube axis (Fig. 5). To assure equal urrival time of the uptical signals at the gated MCP intensifier, the fiber-uptic arrass are cut at different lengths commensurate with the transit time of the photons through the fiber-optic bundles and with the time-offlig: of the elextrons between the sampling beads.

The size and pusition of a relatisistic electron beam inside a wiggler magnetic field of a fresecelectron laser is determined using a tartilum/fluer arrangement in the waveguide. ${ }^{15}$ One end of a fiber-optic bundle is connected to this fluor and the other end to a gated NCP intensifier thal is coupled to a Reticen $100 \times 100$ photodiode array camern. This camera is bocated outside the accelerator shicld wall.

X-ray diagnostics. Tine-resolved x-ray measurements are performed with a Reticen linesr photodiode array camera. Efforts are made to use 7 to 28 linear arrays will 1 to 4 MCP detectors in a microstrip transmossion-line genmetry to observe $x$ ravs of several $\mathrm{keV}$ from a bent-orystal spertrometer ${ }^{16}$ with resolution of a few eV Jacoby ${ }^{3}$ has shown that, compared to termination coupling, MCP intensifiers having a mocostrip transmission-line geometry permit high-speed optical shuttering. The MCP delector shown in Fig. 6 was designed by Eekert." This defector uses seven gold microstrip lines on the input side ( $x$-ray side) of the MCP. An Electrical $1-k V$

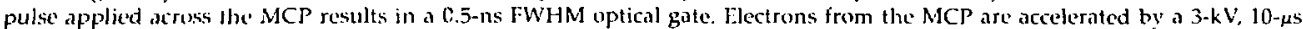
clectrical pulse mbto a P-46 phosphor deposited on a fiber-optic face plate (proxinity-focused diode). To separate the camera from the high-radiation environment near the MCP detector and to increase the signal-to-noise ratio. fiber-nptic arrays couple the P-46 autput corre'sponding to each strip line on! a gated (5kV, $150 \mathrm{~ns}$ ) proximity-focused diode. Coherent fiber-optic bundles then transfer the images to seven Reticon lintar arrays. The arrays are read-out in $1 \mathrm{~ms}$ at $180 \mathrm{Mbit} / \mathrm{s}$.

Gamma-ray and neutron diagnostics. An electronic pinhole neutron experiment uses a gated MCP inlensified Reticon or Fairchild soilid-state camera to study burn efficiencies and implosion symmetries of fission/fusion devices. In the experiment, nuclear radiation is focused through a pintrole onto a plastic fluor. ${ }^{.}$Light from the fluor is then for used onto the camera that digitizes the data und seids them to a memory. These data and the calibration data are processed by computer into a quantized image.

\section{Applications using streak canteras and solid-state cameras}

A Fairchild CCD canera coupled to a MCP intensified streak camera is used for lomegraphy and for time-reselved studies of clectron-lxeam propagation phenumena and nuclear fusion precesses. Table 4 lists the performance characteristics for this şstem.

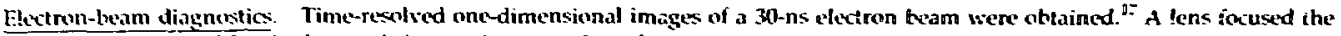
air fluorescence preninced by the beam-dir interaction onte three filner-optic arravs ponstionevi around the beam avis. The fiber-optic

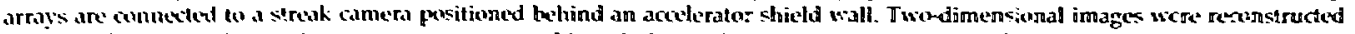

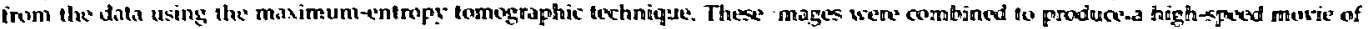
lise clectron-beom pulse propugation in air. 


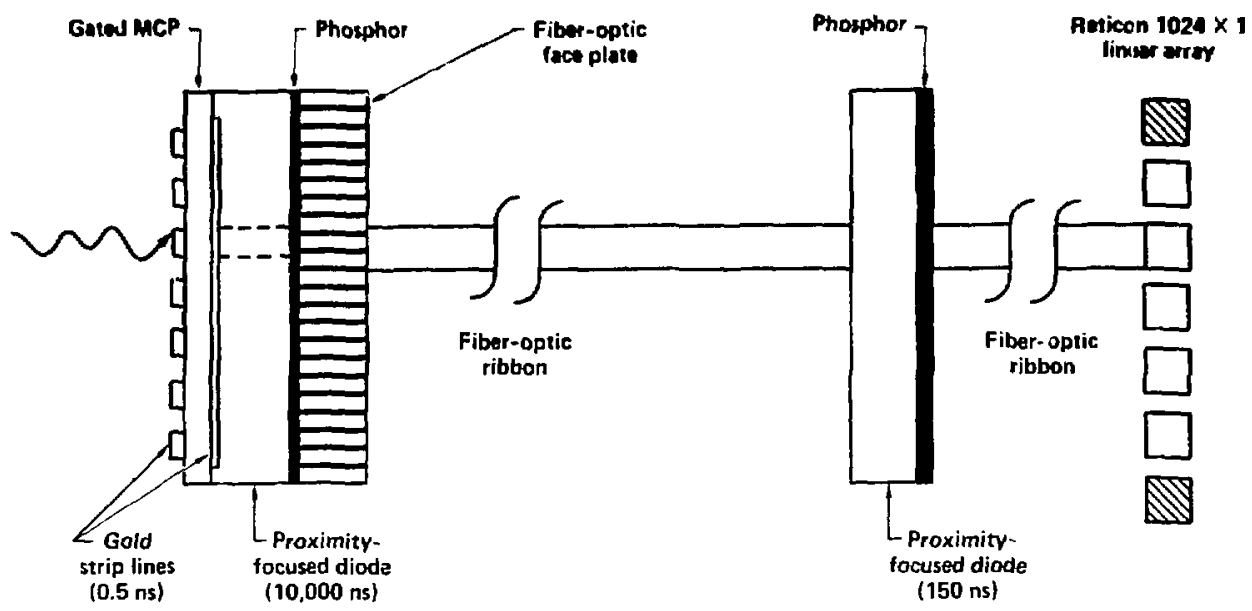

Figure 6. Experimental configuration for measuring time-resolved $x$-ray spectra.

Table 4. Performance characteristics of MCP intensified streak camera/Fairchild CCD system.

\begin{tabular}{|c|c|}
\hline Characteristic & $\begin{array}{l}M C P^{p a} \text { intensified streak camera/ } \\
\text { Fairchild } C C D \text { system }^{b}\end{array}$ \\
\hline Shutter speed (ns) & $0.05-500^{r}$ \\
\hline Resolution $(1 \mathrm{p} / \mathrm{mm})^{\mathrm{d}}$ & 4 \\
\hline Frame time (ms) & 4.9 \\
\hline Dynamic range & 100:1 \\
\hline System gain & 20 \\
\hline Saturation $\left(e \mathrm{rg} / \mathrm{cm}^{2} \cdot \mathrm{s}\right)$ at $570 \mathrm{~nm}$ & $10^{8}-10^{4}$ \\
\hline Digitizer (Mbit/s) & $180^{\mathrm{e}}$ \\
\hline Sensitivity (photoelectron/pixel) & $1^{f}$ \\
\hline
\end{tabular}

\footnotetext{
${ }^{a} 40$-mm-diameter, supplied by ITT

b $244 \times 380$ pixels.

${ }^{\circ} 0.5 \%$ of sweep time, where sweep time is $0.01-100 \mu \mathrm{s}$.

${ }^{\mathrm{A}}$ At $70 \%$ CTF, 250 lines total.

8 bits, parity, overhead.

'At strea:' camera phosphor.
}

Nose-erosion studies of high-current relativistic electron beams have been performed with a system similar to that used for determining electron-beam propagation instabilities. To obtain nanosecond rise-time information, the $\mathrm{MCP}$ was replaced by a streak camera.

Neutron diagnostics. A tomographic system with four slits is used to provide two-dimensional time-resolved images of fusion devices. ${ }^{18}$ A pinhole images the ionizing radiation onto the fluor, and the four slits image sections of the fuor onto four fiber-optic arrays. This system uses a Fairchild CCD and streak camera and a wide-bandwidth fiber-optic transmission system to transfer the unata into the memory.

The streak camera/solid-state camera system is also used for measuring the spatial dependence of temperature in fusion cavities. Essentially, this experiment is similar to the four-slit tomographic arrangement except that the slits are replaced by a lens.

\section{Conclusions}

Time-resolved imaging is an important diagnostic of pulsed radiation sources at LLNL_in the laboratory as well as in the field. Brih the MSCP intensified solid-state two-dimensional framing cameras and the streak camera/solid-s!zte carriera systern are cumplementary.

The framing camera pravides for snap shots with high spatial resolution. in geneial. these cameras are cafable of $10^{5}$ spatial points, 2-ns cptical gate time, 2-ms frame time, and 200.1 dirnamic range. 
The streak eamera system provides for limited spatial points each with high teniporal resolution. These systems are generally characterized by 250 spatial points, 0.05-ns temporal resolution, 5-ms frame time, and 100:1 dynamic range.

New cameras are being developed to meet the demand for higher temporal and spatial resolution, faster frame time, larger dynamic range, and better data processing and data archiving systems. Fast diagnostic systems that use the Reticon $256 \times 256$ and $1024 \times 1$ photodiode arrays h.ve been developed and incorporated into experiments. Work continues on studying low-resistivity photocathode and low-impedance high-voltage pulsers for fast gateable MCP.

\section{References}

1. Lear, R., "Fast Imaging Applications in the Nucleas Test Program," in IEEE Trans. Nuclear Science, Feb. 1984, Vol. NS-31 (1), pp. 495-503.

2. Koehler, H. A., and Cornish, J. P., MCP intensifier charactesization Lawrence Livermore National Laboratory, Livermore,

CA, ATA Note 192, private communication (Jan, 11, 1983).

3. Jacoby, B. A., Kotecki, D. E., and Lear, R. D., "Direct Gating of Microchannel Plates," IEEE Trans. Nucl. Sci., Vol. NS-30 (6), pp. 4624-4627, Dec. 1983.

4. Kotecki, D. E, and Lear, R., "Optical shutters using microchannel plate (MCP) intensifier tubes," in Proc. Soc. Photo-Optical Instrumentżion Engineers, 1983, San Diego, CA, Aug. 1983 (SPIE, Bellingham, WA), Vol. 427, pp. 62-78, 1983.

5. Yates, G. L., King, N. S. P., Jaramillo, S. A., Noel, S. W., Globy, P. L., Aeby, I, and Detch, J. L., "Nanosecond Image Shuttering Studies at Los Alamos National Laboratory," in IEEE Trans. Nuclear Science, Feb. 1984, Vol. NS-21, pp. 484-489.

6. Yates, G. L., King, N. S. P., Jaramillo, S. A., Ogle, J. W., and Noel, B. W., "Image shutters: gated proximity focused microchannel plate (MCP) wafer tubes versus gated silicon intensifier target (SIT) vidicons," in Proc. 15th International Congress on High-Speed Photography and Photonics, 1982 (SPIE, Bellingham, WA), Vol. 348, pp. 422-433, 1982.

7. Yates, G. L., Ogle, J. W., and King, N. S. P., "Overview of pulses for nanosecond gating of image shutter tubes," in Proc. 15th International Congress on High-Speed Photography and Photonics, 1982 (SPIE, Bellingham, WA), Vol. 348, pp. 434-437, 1982.

8. Li, K. K., Whinnery, J. R., and Dienes, A., "Optical switches for generation and pulse shaping of ultrashort electrical pulses," Picosecond Lasers and Applications, 1982 (SPIE, Bellingham, WA), Vol. 322, pp. 124-130, 1982.

9. Lee, C. H., "Picosecond Optoplectronic Switcl.ing in GaAs," Appl. Phys. Lett., Vol. 30 (2), pp. 84-86, 1977.

10. Sammons, T. J., EL PINEX Handbook, EG\&G/SRO, San Ramon, CA, EGG1183-4229 (Jan. 1982).

11. Tseng, H., and Lin, C. "A High Frame Rate RA $256 \times 256$ Solid State Image Sensor," Electronic Imaging '84, Boston, MA Sept. 11-13, 1984 (Society of Photographic Scientists and Engineers, Springfield, VA).

12. O'Brien, C. R., Lawrence Livermore National Laboratory, Livermore, CA, private communication (1984).

13. Koehler, H. A., and Clendener L., Fairchild CCD-2000 camera characterization, Lawrence Livermore National Laboratory, Livermore, CA, ATA Note 196, private communication (Jan. 13,1983).

14. Stephenson, P. S., Lawrence Livermore National Laboratory, Livermore, CA, private communication (1984).

15. Orzechowski, T. J. Koehler, H. A., and Edwards, W. "A novel probe for determining the size and position of a relativistic electron beam," in Proc. Soc. Photo-Optical Instrumentation Engineers, San Diego, CA, Aug. 19-24, 1984 (SPIE, Bellingham, WA).

16. Eckert, M., and O'Brier, C. R., Lawrence Livermore National Laboratory, Livermore, CA, private communication (1984).

17. Koehler, H. A., Jacoby, B. A., and Nelson, M., Time-resolved tomographic images of a relativistic electron beam," in Proc. Soc. Photo-Optical Instrumentation Engineers, San Diego, CA, Aug. 19-24, 1984 (SPIE, Bellingham, WA).

18. Jacoby, B. A., Lawrence Livermore National Laboratory, Livermore CA, private communication (1984).

\section{Acknowledgments}

We thank the following people from LLNL for their contributions: Richard Lear, Barry Jacoby, Mark Eckert, Hal Schulte, Charles O'Brien, Paul Stephenson, and Lamar OIk. Mauro Fardo and Tim Sammons from EG\&G/SRO also supplied information for this paper. This work was performed under the auspices of the U.S. Department of Energy by ${ }^{\prime}$; wrence Livermore National Laboratory under Contract W-7405-Eng-48. 